\title{
Factor analysis study on sexual responses in women with Type 2 diabetes mellitus
}

\begin{abstract}
Aims: This study compared the components of sexual responses between Malaysian women with Type 2 diabetes mellitus and those without the disease.

Methods: This cross-sectional study measured sexual responses by using the validated Malay version of Female Sexual Function Index. A factor analysis with varimax rotation method was employed using principal component analysis to explore the correlation structure of the different domains of sexual responses between the two groups. Components of sexual responses were obtained using Kaiser's criteria and compared between those in the diabetic and non-diabetic groups.

Results: A total of 353 women (178 with diabetes and 175 without diabetes) were recruited. Three components of sexual responses emerged from the analysis in the study and control groups. Sexual pain was found to form a component together with lubrication and orgasm domains among the women with diabetes, unlike those without diabetes, where pain stood on its own. Sexual desire and arousal formed one component and satisfaction formed another in both groups.

Conclusions: The domains in the sexual responses of Malaysian women were highly overlapping. It is concluded that the presence of pain as part of lubrication and orgasm component in women with diabetes indicates the importance of intact genital sensation, even though an adverse type of sensation, for vaginal congestion and orgasm to occur in this group of women.
\end{abstract}

Keyword: Sexual responses; Women; Type 2 diabetes mellitus 